\title{
La petite propriété paysanne en République : les incertitudes du discours républicain
}

La pertinence économique de la politique agricole des fondateurs de la Troisième République a fait l'objet de nombreux débats. Elle a longtemps suscité la désapprobation des chercheurs : pour des raisons électorales et idéologiques, les républicains auraient cherché avant tout à protéger la petite exploitation familiale, au risque de retarder durablement la modernisation de l'agriculture française ${ }^{1}$. Mais depuis une trentaine d'années, des jugements plus nuancés se sont fait jour : l'efficacité économique des petites unités de production est souvent admise ${ }^{2}$ et leur sauvegarde a même été parfois présentée comme indispensable au développement industriel, commercial et financier des sociétés modernes ${ }^{3}$.

En revanche, le socle de principes et de convictions politiques sur lequel reposent les actions des républicains en faveur de l'agriculture n'a jamais vraiment été discuté. Leurs positions à cet égard paraissent certes d'emblée d'une grande cohérence. Habités par un idéal politique et social égalitaire, où l'indépendance économique affranchirait l'individu des relations de domination que la loi n'avait pas suffi à défaire, ils se montraient également soucieux de répondre aux aspirations de la majorité de la population, les ruraux, toujours avides d'acquérir le moindre lopin de terre. Comment n'auraient-ils pas dès lors été acquis à la défense de la petite exploitation rurale et plus précisément de la petite propriété paysanne ? ? $^{4}$ Les éloges unanimes qu'ils réservent à la réfutation du socia-

1. Voir par exemple Gabriel DÉSERT, «Bilan économique à la veille de la Première Guerre mondiale ", in Georges DUBY, Armand Wallon (dir.), Histoire de la France rurale, Paris, Le Seuil, 1976, tome 3, pp. 429-443.

2. Voir par exemple Jean-Luc MAYAUD, La petite exploitation triomphante : France, XIX siècle, Paris, Belin, 1999.

3. C'est la thèse de Claude SERvolin, "Les politiques agricoles ", in Madeleine GrawitZ, Jean LECA (dir.), Traité de science politique, Paris, PUF, 1985, tome 4, pp. 155-260.

4. Serge BERSTEIN, "La politique sociale des républicains", in Serge BERSTEIN, Odile RUDELLE (dir.), Le modèle républicain, Paris, PUF, 1992, pp. 189-208. 
lisme agraire que Paul Deschanel oppose en 1897 à Jean Jaurès devant la Chambre des députés sont à ce titre souvent invoqués en exemple ${ }^{5}$ : les républicains de toutes tendances apparaissent alors soudés dans la glorification de la petite propriété foncière, qui semble là revêtir sa forme la plus aboutie.

C'est pourtant occulter les débats qui divisent le camp républicain sur la question depuis 1848 et qu'on voudrait ici retracer à travers la presse et les discussions parlementaires, en se concentrant surtout sur quatre moments particulièrement éclairants : le conflit suscité par les 45 centimes en juin 1848, les interrogations soulevées par le bonapartisme rural autour de 1860 , les réactions au discours prononcé par Ferry à Bordeaux en août 1885, le grand débat Jaurès/Deschanel en juin et juillet 1897. Ils permettent en effet de mettre en lumière les incertitudes qui s'accumulent tout au long du second $\mathrm{XIX}^{\mathrm{e}}$ siècle à propos de la viabilité économique et politique de la petite propriété et qui, au-delà de la définition de la politique agricole, ébranlent profondément le projet républicain dans son ensemble. La véhémence avec laquelle les républicains avancent et discutent les chiffres des enquêtes agricoles renvoie d'abord à une interrogation sur les besoins et aspirations de la grande majorité du peuple français et donc sur les contours d'un programme véritablement républicain, c'est-à-dire attentif aux intérêts du plus grand nombre. Elle témoigne aussi et surtout d'une inquiétude à l'égard des évolutions économiques du moment, qui mettent en question leur capacité à réaliser les promesses de la Révolution française, identifiées à l'affranchissement du paysan et au morcellement du sol. L'enjeu est majeur : ces hommes qui ont toujours associé la République au progrès ne peuvent concevoir sans appréhension l'éventualité d'une contradiction entre les lois économiques et leurs convictions politiques et sociales.

\section{La République au chevet des petits propriétaires terriens}

\section{La petite propriété foncière : un idéal social équivoque dès 1848}

1848 inaugure pour le camp républicain une longue série d'interrogations sur la petite propriété foncière. Le débat sur le morcellement du sol n'est alors pas nouveau - il agite les milieux savants et poli-

5. Pierre BARral, Les agrariens français de Méline à Pisani, Paris, Armand Colin, 1968, p. 164 et suiv. 
tiques depuis le milieu du XVIII ${ }^{\mathrm{e}}$ siècle - et les républicains y ont toujours défendu la petite propriété paysanne. Mais l'avènement de la Seconde République et avec elle l'instauration du suffrage universel masculin les obligent à préciser leurs positions.

La chose est d'autant plus urgente que la République est peu soutenue dans les campagnes: les élections d'avril 1848 n'ont pas donné partout satisfaction aux républicains, la levée de l'impôt des 45 centimes, décidé au printemps pour combler le déficit public, rencontre des difficultés dans certains départements ruraux, et l'échec des candidats républicains devant Louis-Napoléon Bonaparte à la présidence de la République démontre bientôt la défiance des populations rurales à l'égard des artisans du nouveau régime. La détresse des campagnes est en outre exploitée par la droite, mais aussi par les socialistes, qui invoquent les difficultés de la petite propriété foncière à l'appui de leurs revendications : collectivisation des terres ou plus fréquemment colonisation agricole de la France, sous la forme d'ateliers gérés par l'État ou au contraire au sein d'associations autonomes.

Face à ces nombreux défis, les républicains réaffirment d'abord leur foi dans la petite propriété. Le discours très applaudi que François-Joseph Ducoux oppose en juin 1848 au socialiste Pierre Leroux, qui vient de faire un tableau des plus noirs de l'agriculture française, en rappelle les grandes lignes : « la liberté n'a surgi en France que le jour où il a été permis au paysan d'acheter la parcelle de terre sur laquelle il vivait en serf", il faut donc "développer en France le sentiment honnête de la propriété » si l'on veut " rendre la République impérissable ».

Mais cet idéal commun cache des divergences de vues que les difficultés de la République rendent bientôt manifestes. Le conflit suscité dans le camp républicain par l'impôt des 45 centimes en donne un bon aperçu. Pour les républicains modérés, la mesure s'impose. Elle a en effet l'avantage à leurs yeux de répondre aux "nécessités » du moment - le mot revient fréquemment dans leur argumentation ${ }^{7}-$ tout en respectant les principes du nouveau régime : elle pèse avant tout sur la propriété

\section{Séance du 16 juin 1848, Moniteur universel, 17 juin 1848, p. 1402.}

7. Voir notamment l'intervention du ministre des finances, Charles Duclerc, en réponse aux interpellations sur les 45 centimes lors de la séance du 17 juin 1848 , à la Chambre: Moniteur universel, 18 juin 1848, p. 1418. Voir aussi les argumentaires de Lamartine et de Garnier-Pagès: Alphonse de LAMARTINe, Histoire de la révolution de 1848, Paris, Perrotin, 1849, tome 2, p. 105 ; Louis-Antoine GARNIER-PAGÈS, Un épisode de la Révolution de 1848 : l'impôt des 45 centimes, Paris, Pagnerre, 1850. 
foncière, qui reste ainsi au fondement de l'ordre social et politique ${ }^{8}$, mais épargne les plus modestes en vertu de nombreuses exonérations, au nom de la justice sociale. Les républicains modérés n'y voient donc aucune contradiction avec leur idéal social et politique : la République ne doit pas selon eux bouleverser le système social établi mais seulement encourager la division déjà bien avancée de la propriété en France pour parfaire les conditions matérielles du nouvel ordre politique : seule une nation de petits propriétaires terriens était susceptible de développer l'esprit d'indépendance nécessaire au bon fonctionnement des institutions démocratiques.

Quoiqu'ils aspirent également, et pour les mêmes raisons politiques que les modérés, à multiplier les petits propriétaires dans le pays, les radicaux donnent un tout autre sens à cet idéal politique et social. La diffusion de la petite propriété incarne à leurs yeux une organisation sociale nouvelle, qu'il n'est possible d'instaurer qu'en rompant résolument avec le passé. Or les 45 centimes non seulement renouent avec les principes fiscaux des précédents régimes, eux aussi fondés sur la propriété foncière, mais en ponctionnant l'épargne des petits paysans, ils les empêchent en outre d'acquérir un lopin de terre ou d'arrondir leur parcelle. Ils deviennent ainsi pour eux le symbole de la "République bourgeoise ", qu’ils opposent à la "vraie République, la République démocratique et sociale " $"$, dont le programme agricole est désormais mieux connu ${ }^{10}$ : modification du système fiscal, prise en charge par l'État des assurances, création de caisses de secours, organisation du crédit agricole, autant de mesures qui visent à favoriser l'accès des plus humbles à la propriété.

Le conflit sur la signification à donner à la défense de la petite propriété n'est pas purement théorique. À l'heure du suffrage universel, il constitue un enjeu à la fois électoral et idéologique. L'électorat paysan majoritaire est en effet érigé en arbitre, dans la conquête du pouvoir mais aussi dans la validation des propositions des uns et des autres : pour les

8. Ce que confirme Robert SCHNERB, "Les hommes de 1848 et l'impôt ", in Jean BOUVIER, Jacques WOLFF (dir.), Deux siècles de fiscalité française XIX et XX siècle : histoire, économie, politique, Paris, La Haye, Mouton, 1973, pp. 105-157.

9. Félix PYAT, «Toast aux paysans, porté au banquet du 24 février 1849 », in Eugène SuE, Le Républicain des campagnes, Paris, Libraire de la Propagande démocratique et sociale européenne, 1851, p. 68.

10. Annie BLETON-Ruget, "Aux sources de l'agrarisme républicain : la propagande démocrate-socialiste et les campagnes (1848-1851) ", Cahiers d'histoire, $\mathrm{n}^{\circ} 2$, tome 43, 1998, pp. 283-299. 
républicains, le suffrage universel ne se réduit pas à une procédure électorale, il permet surtout de faire entendre la voix infaillible du peuple, qui choisit ainsi ses véritables porte-parole. Au début de la Seconde République, les radicaux semblent avoir - très provisoirement - l'avantage : aux élections législatives de 1849, les « démocs-socs " arrivent en tête dans de nombreux départements ruraux. La suite des événements les contraint à s'effacer, mais ces premières victoires ont suffi à ancrer durablement dans la mémoire républicaine l'idée selon laquelle la Seconde République aurait péri de n'avoir pas pris suffisamment en compte les aspirations de la paysannerie.

\section{La statistique comme champ de bataille}

Mises en sourdine sous le Second Empire puis dans la conquête difficile de la Troisième République, les divisions internes au camp républicain ressurgissent dès qu'il accède au pouvoir, à la fin des années 1870 . Elles n'épargnent pas l'idéal du petit paysan propriétaire, qui semble déchiré par les mêmes contradictions qu'en 1848 : quand les opportunistes y voient la consolidation des acquis de 1789, les radicaux en attendent toujours la réalisation du versant social de la Révolution française, jusqu'ici négligé au profit de sa dimension purement politique.

Le conflit s'est pourtant déplacé. En 1848, il avait été avant tout porté par la question des mesures à adopter pour résoudre le malaise des populations rurales, qui semblait quant à lui indiscutable. Ainsi, lorsque le socialiste Pierre Leroux avait tenté en juin 1848 d'amener les représentants du peuple sur le terrain des "faits ", pensant par là les convaincre scientifiquement des bienfaits de l'association agricole, il n'avait cessé d'être interrompu par des élus soucieux d'en venir au débat de principe : "Nous sommes tous d'accord sur l'existence du mal, mais veuillez nous en indiquer le remède ! " ${ }^{11}$

À partir des années 1880, c'est en revanche dans la discussion sur les faits que se joue le conflit. Pour les opportunistes, la France est déjà une nation de petits propriétaires terriens, et c'est d'ailleurs ce qui fait la force de la République à leurs yeux : "C'est là une force immense, affirme Jules Ferry dans un discours prononcé à Bordeaux en 1885, et sur laquelle repose la sécurité de notre société, que cette population de petits propriétaires, si nombreux qu'ils constituent à eux seuls la majorité du

11. Ici, Antony Thouret. Séance du 17 juin 1848, Moniteur universel, 18 juin 1848, p. 1417 et suivantes. 
nombre dans la nation $»^{12}$. Une telle affirmation est intolérable aux radicaux, qui n'ont de cesse de souligner au contraire les difficultés dans lesquelles se débattent la plupart des paysans : il leur paraît exorbitant de donner le nom de propriétaires aux détenteurs d'un lopin de terre minuscule, croulant de surcroît bien souvent sous la dette hypothécaire. Le passage du discours de Bordeaux cité plus haut fait ainsi l'objet de violentes attaques dans le journal de Clemenceau, La Justice: "Il choisit de préférence les statistiques les plus anciennes, les plus fausses, les plus controuvées, s'indigne Charles Longuet en première page. Proclamer dans un discours-ministre que les petits propriétaires sont si nombreux en France "qu'ils constituent à eux seuls la majorité du nombre dans la nation", c'est dépasser les bornes même de la plaisanterie permise à nos hommes d'État. La statistique montrait, il y a plus de vingt ans, que trois millions de prétendus propriétaires campagnards étaient exempts de la cote personnelle. Ils étaient des pauvres, ces propriétaires! »"

L'influence du positivisme et la sociologie naissante les conduisent en effet à revendiquer pour leur doctrine la conformité aux lois sociales déduites de l'"observation": le terme occupe une place centrale dans le discours de Ferry comme dans l'article de Longuet. Si les paysans sont effectivement dans leur grande majorité des petits propriétaires indépendants, alors le conservatisme des opportunistes est ce dont le pays a besoin. Mais s'ils végètent au contraire dans la dépendance économique, ce sont en revanche les radicaux qui détiennent la formule de la "vraie République " ${ }^{14}$. "Vous dites que le paysan veut garder la terre, lance ainsi Longuet à Ferry. Parbleu! Nous autres, radicaux, nous allons encore plus loin dans votre voie, nous ajoutons qu'il veut aussi l'acquérir " ${ }^{15}$.

Si la configuration du débat a changé depuis 1848 , l'enjeu est donc identique : derrière la bataille des chiffres se joue un conflit de légitimité que le peuple est appelé à trancher dans les élections. Et au début de la Troisième République, c'est l'opportunisme qui semble avoir le soutien

12. "Discours de Bordeaux, du 30 août 1885 ", Discours et opinions de Jules Ferry, Paris, Armand Colin, 1898, tome VII, p. 41.

13. Charles LongueT, "Avocasserie ", La Justice, $1^{\text {er }}$ septembre 1885, p. 1.

14. Daniel Mollenhauer a souligné l'importance de cet enjeu à la fin du XIX siècle : Daniel Mollenhauer, Auf der Suche nach der "wahren Republik» : die französischen "radicaux" in der frühen Dritten Republik, 1870-1890, Bonn, Bouvier, 1997.

15. Ibid. 
de la majorité rurale ${ }^{16}$. Comme Marx quelque vingt ans plus tôt ${ }^{17}$, les radicaux sont alors conduits à distinguer les aspirations de la paysannerie de ses véritables intérêts, dont ils affirment être les seuls représentants sincères. Le clivage semble avoir un rôle structurant à gauche. Une dizaine d'années plus tard, Jaurès développe une argumentation du même ordre, mais cette fois au profit du socialisme. Les chiffres qu'il invoque à la tribune en 1897 lui servent à démontrer l'«agonie » inéluctable de la petite propriété $^{18}$ - réalité économique qui confirme selon lui la validité des conceptions défendues par les socialistes et fait de ces derniers les porte-parole tout désignés de la majorité rurale, quoiqu'elle n'en soit pas elle-même encore tout à fait consciente :

«Et peu nous importe que ces hommes, de longtemps encore, ne puissent nous entendre et nous suivre; peu nous importe qu'à la merci de ceux qui dispensent le travail et le salaire, ils soient destinés longtemps encore peut-être, métayers ou journaliers, à consacrer de leur vote passif précisément la servitude et la misère que nous voudrions faire cesser $(A p$ plaudissements à l'extrême gauche - réclamations au centre et à droite). Notre rêve de justice n'est pas d'un jour ; notre œuvre n'est pas une combinaison éphémère d'intérêt prochain, et c'est d'abord aux plus dépendants, aux plus obscurs, aux plus dénués que va notre sollicitude, précisément parce qu'ils sont les plus obscurs, les plus dépendants, les plus dénués!" ${ }^{19}$

Le glissement à gauche fut d'ailleurs bien perçu par les radicaux, qui se sentirent en quelque sorte "doublés " par le socialisme agraire. Quelques années plus tard, un discours de Compère-Morel provoque ainsi l'indignation d'Adolphe Girod: " Mais c'est tout le programme radical ! Nous avons commencé avant vous! ${ }^{20}$

16. Les élections de 1885 sont en revanche confuses. Pour Odile Rudelle, le succès des radicaux n'est qu'un leurre permis par le mode de scrutin : "En dépit des apparences, [les opportunistes] correspondent au sentiment intime du pays ». Odile RUDELLE, La République absolue : aux origines de l'instabilité constitutionnelle de la France républicaine 1870-1889, Paris, Publications de la Sorbonne, 1982, p. 157.

17. Karl MARX, Le dix-huit brumaire de Louis Bonaparte, Paris, Messidor/Éditions sociales, 1984 ( $1^{\text {ère }}$ édition 1852).

18. Séance du 26 juin 1897, Journal officiel de la République française, 27 juin 1897, p. 1692.

19. Séances du 19 juin 1897, Journal officiel de la République française, 20 juin 1897, p. 1588.

20. Séance du 6 décembre 1909, Journal officiel de la République française, 7 décembre 1909, p. 3199. Cité par Pierre BARRAL, Les agrariens français..., op. cit., p. 165. 


\section{L'héritage disputé de la Révolution française}

\section{La hantise des nouvelles féodalités économiques}

Les conceptions économiques des radicaux n'avaient cependant rien à voir avec celles des socialistes. Ils restaient en effet convaincus que les lois économiques et sociales qui présidaient au mouvement de la propriété en France favorisaient le morcellement, tandis que leurs adversaires socialistes affirmaient au contraire le caractère inexorable de la concentration, qu'ils voyaient déjà pleinement à l'œuvre dans l'industrie et dont ils croyaient deviner les prémices dans l'agriculture.

Cette conviction, héritée des idéaux révolutionnaires de 1789, avait longtemps uni les républicains de toutes tendances. Sous la Seconde République, au-delà des divergences évoquées plus haut, modérés et radicaux s'accordaient ainsi sur l'adéquation profonde de l'économie et du politique. L'avènement précoce de la démocratie en France leur paraissait en effet directement lié à la division des patrimoines, dans l'industrie comme dans l'agriculture. Le modéré François Ducoux l'avait rappelé à la tribune contre les doctrines socialistes de Pierre Leroux : "N'est-il pas vrai qu'à mesure que la petite industrie s'est créée en France, qu'à mesure que la grande propriété s'est morcelée, la liberté a surgi sur notre territoire? " ${ }^{21}$ Ledru-Rollin, qui incarne pourtant un républicanisme beaucoup plus avancé, ne dit pas autre chose : "La distribution de la propriété foncière ", écrit-il en 1850, est "si capitale dans le régime des peuples, qu'elle suffirait presque à elle seule pour déterminer la forme de leurs institutions $"{ }^{22}$. Pour l'un comme pour l'autre, l'Angleterre constitue donc un contre-modèle : la démocratie, qui leur semble fleurir naturellement en France, serait impossible outre-Manche, tant la concentration des propriétés y est poussée.

Évoquer l'idée selon laquelle l'économie pourrait bien être au contraire régie par des lois de concentration était alors insupportable aux républicains. C'était admettre et légitimer le retour de l'Ancien Régime : la «concentration" était pour eux synonyme de "féodalité économique ", qui ramènerait en France la féodalité politique et ruinerait ainsi l'œuvre de la Révolution française. S'il y avait en 1848, dans l'industrie

21. Séance du 16 juin 1848, Moniteur universel, 17 juin 1848, p. 1402.

22. Alexandre LEDRU-Rollin, De la décadence de l'Angleterre, Paris, Escudier frères, 1850, tome 1, p. 25. 
notamment, des signes de concentration, c'était selon eux le fait des gouvernements monarchiques précédents, qui avaient cherché à freiner l'émancipation de la nation. Lorsque Ferdinand Flocon, alors ministre $\mathrm{du}$ Commerce et de l'Agriculture, monte à la tribune pour présenter les projets agricoles du gouvernement provisoire, il déplore ainsi que «la France a[it] été jetée d'une manière anormale dans la voie des spéculations ${ }^{23}$, associant la misère ouvrière au retour en arrière quasi pathologique - «anormal » - que constitue à ses yeux la concentration financière et industrielle.

Mais là encore, le second $\mathrm{XIX}^{\mathrm{e}}$ siècle voit les républicains se diviser. Les premières inquiétudes apparaissent sous le Second Empire, dans les années 1860. Un texte de Ferry, rédigé vers 1862 mais resté alors inédit, illustre bien les évolutions que subissent les conceptions économiques des républicains les plus libéraux. Après avoir rappelé le «fonds d'idées économiques et sociales » qui unit les républicains depuis 1789 autour de la défense de la petite propriété industrielle et agricole, Ferry invite ses lecteurs à prendre conscience de leur erreur d'appréciation : «Le temps est venu pourtant de réagir contre un fétichisme banal. L'esprit moderne peut, sans s'amoindrir, devenir scientifique, après avoir été révolutionnaire. Il nous est permis de jeter sur les problèmes sociaux un regard plus libre que nos pères, et de nous croire, sans vanité, plus de lumières, ayant de plus qu'eux la leçon de trois quarts de siècle d'expérience. " ${ }^{24}$.

La révision ne porte pas sur le morcellement agricole, qui lui paraît encore "triomphant " ${ }^{25}$, mais sur l'état de l'industrie, qui, loin de se diviser, offre un terrain privilégié aux "agglomérations du capital ${ }^{26}$. Ferry refuse d'y voir un phénomène anormal porté par les forces de la réaction : il s'agit selon lui d'un "grand fait ", d'une "tendance " si forte qu'elle se joue de l'action des gouvernements ${ }^{27}$.

Pour un républicain comme Ferry, le constat a quelque chose d'effrayant : sa foi dans la science lui impose de reconnaitre la nécessité de la concentration économique, qu'il considère pourtant comme despotique. La «féodalité industrielle » rappelle ainsi la noblesse territoriale et

23. Séance du 17 juin 1848, Moniteur universel, 18 juin 1848, p. 1416.

24. "La concentration industrielle", Discours et opinions de Jules Ferry, op. cit., pp. 516-517.

25. Ibid.

26. Ibid., p. 519.

27. Ibid., pp. 501-502. 
les monopoles royaux que les révolutionnaires de 1789 voulaient justement détruire : "entre l'Ancien Régime et la société moderne, n'est-ce pas la chaîne rompue qui se renoue? " ${ }^{28}$.

\section{Réaliser les promesses de la Révolution française}

Le remède est à chercher pour Ferry dans l'exemple anglais, que les quarante-huitards avaient condamné mais qui, sous le Second Empire, en est venu à incarner la liberté aux yeux de nombreux républicains : ce que montre l'expérience de l'Angleterre selon lui, c'est que la liberté politique largement répandue devient " un merveilleux contrepoids au despotisme des grands capitaux ${ }^{29}$. Ferry remanie ainsi profondément l'idéal républicain. Il renonce en effet aux représentations unitaires qui marquaient jusqu'ici le discours républicain - une même loi d'individualisation était supposée à l'œuvre dans tous les domaines, économiques, sociaux ou politiques - pour défendre au contraire l'idée d'un monde complexe, où l'agriculture et l'industrie sont régies par des lois différentes (morcellement ou concentration), qui n'intègrent le mouvement du progrès qu'à condition d'être contrebalancées par le perfectionnement des institutions politiques léguées par la Révolution française.

Ce sont ces conceptions qui, sous la Troisième République, guident ensuite les opportunistes lorsqu'ils accèdent au pouvoir, au grand dam des radicaux, qui les considèrent pour leur part en totale contradiction avec l'esprit de 1789. Réagissant au discours tenu par Ferry à Bordeaux en 1885, Longuet est ainsi ulcéré de le voir rappeler, comme si les radicaux l'avaient " oublié », que le paysan français « ne s'est attaché passionnément à la cause de la Révolution française " que parce qu' " elle lui a donné la possession incontestée et souveraine " de sa terre ${ }^{30}$. C'est en effet revendiquer indûment à ses yeux l'héritage de la Révolution française : «Mais vous, que pouvez-vous faire pour assurer au paysan ces conquêtes matérielles qui l'attacheraient aussi indissolublement à la République que le Code civil l'attacha à la Révolution française! Rien, rien, rien! Vous ne pouvez rien parce que vous ne toucherez jamais à l'oligarchie économique qui vous enserre. " ${ }^{31}$

28. Ibid., p. 520.

29. Ibid., p. 535.

30. «Discours de Bordeaux, du 30 août 1885 », Discours et opinions de Jules Ferry..., op. cit., p. 41.

31. Charles Longuet, «Avocasserie », La Justice, art. cit., p. 1. 
Pour les radicaux, attachés à l'idéal d'une nation de petits producteurs, qu'ils soient agricoles ou industriels, les opportunistes font preuve d'une grande hypocrisie lorsqu'ils invoquent la Révolution : ce sont eux qui la trahissent ! En acceptant la concentration industrielle et financière, ils dissocient le destin des différentes branches de l'économie, qui, interdépendantes, devraient être aux yeux des radicaux subordonnées à la même loi de morcellement, ils ouvrent la voie au retour, sous une nouvelle forme, des relations de domination que les révolutionnaires de 1789 visaient à renverser.

Les débats sur l'état de la petite propriété foncière mettent donc en jeu des passions qui vont bien au-delà des questions de pure politique agricole : les républicains de tous bords y voient en effet un enjeu majeur pour la Troisième République - la fidélité à l'événement fondateur que représente pour eux la Révolution française et à partir duquel ils évaluent toute prétention à définir la "vraie République »"

Jaurès l'a bien perçu, qui, en 1897, se réclame à son tour de 1789 : " Pour préciser l'œuvre révolutionnaire nouvelle qu'accomplira, en ce qui concerne la propriété de la terre, le socialisme triomphant, il m’est facile, affirme-t-il dans la séance du 3 juillet, de me reporter aux traditions, aux formules mêmes, aux principes et aux procédés de cette révolution française que, sans cesse, vous revendiquez contre nous ${ }^{33}$.

Son argumentation pourrait aujourd'hui paraitre peu convaincante. Elle prend appui sur la nationalisation des biens du Clergé décrétée en novembre 1789, qui incarne selon Jaurès le véritable esprit de la Révolution. Mais les révolutionnaires, ajoute-il, se sont ensuite fourvoyés lorsqu'ils ont procédé à la vente de ces biens, puisque cette dernière a avant tout profité à la bourgeoisie, au détriment des paysans. Il peut alors conclure qu'un siècle plus tard, le socialisme est le seul qui puisse, grâce à la nationalisation de la propriété foncière, réaliser les promesses de la Révolution française : «Il ne procédera pas à ces partages, il ne donnera pas la terre à qui pourra l'acheter, car les classes dépouillées ne sont pas en mesure d'acheter les bénéfices du régime nouveau " ${ }^{34}$.

32. Daniel Mollenhauer a montré combien cette question était alors prégnante dans les discussions sur les institutions. On voit ici que les débats sur la politique agricole ont aussi constitué un haut lieu de la " recherche de la vraie République ».

33. Séance du 3 juillet 1897, Journal officiel de la République française, 4 juillet 1897, p. 1808.

34. Ibid., p. 1809. 
Le raisonnement a cependant dû être crédité d'une certaine efficacité par Deschanel, qui consacre un long passage de son discours non seulement à le réfuter, mais aussi à le retourner contre les socialistes. Avant de rappeler, citations à l'appui, le rôle central accordé par les révolutionnaires à la propriété privée, il accuse en effet le socialisme de travailler au retour de l'Ancien Régime: "Tout cet Ancien Régime enfin, qui n'est que l'histoire des usurpations continuelles du pouvoir contre la propriété [...] que la Révolution française a eu la gloire d'abolir une fois pour toute [...] - c'est cela que vous voulez faire revivre au profit du Gouvernement de demain - le vôtre! » ${ }^{35}$. Accomplissement de la Révolution française versus retour de l'Ancien Régime : à la fin du XIX siècle, l'opposition, qui semble n'avoir pas perdu sa force de persuasion, ressurgit immanquablement dès qu'il s'agit de petite propriété foncière.

\section{Le progrès en question}

\section{Les contradictions de la petite propriété foncière}

Inscrire la République dans la tradition de 1789 ne suffit pourtant pas aux yeux de ses fondateurs. Ils sont également convaincus qu'il leur faut aussi préparer l'avenir, en identifiant la République au progrès. Longtemps, ces deux impératifs ont paru n'en faire qu'un. Jusqu'en 1848 on l'a vu, les idéaux révolutionnaires semblaient marcher de concert avec le progrès économique et social : dans l'esprit des républicains, la division croissante des fortunes soutenait les progrès de la démocratie dans le pays, qui en retour venait consolider la petite propriété. Le morcellement $\mathrm{du}$ sol apparaissait ainsi comme la marque d'une nation en voie d'émancipation économique, sociale et politique.

À partir de 1848, il leur semble au contraire de plus en plus difficile de nouer ensemble l'héritage du passé révolutionnaire et les exigences du progrès. La petite propriété foncière reste pour eux le legs fondateur de la Révolution française, qui, en donnant la terre aux paysans, en a fait des hommes et des citoyens. Mais ils doutent bientôt de son identification au progrès économique, social et politique. Les petits propriétaires terriens ne constituent-ils pas le cour de l'électorat qui, depuis qu'il dispose du suffrage universel, accorde immanquablement son soutien aux adversaires

35. Séance du 10 juillet 1897, Journal officiel de la République française, 11 juillet 1897, p. 1940. 
de la République? L'argumentation peu à peu élaborée par les républicains pour expliquer leurs échecs électoraux dans les campagnes accuse en effet la petite propriété : cette dernière isolerait ses détenteurs de tout contact avec autrui, le tenant ainsi éloigné des nouvelles idées, qu'elles soient techniques ou politiques.

On sait désormais combien ce constat était loin de la réalite ${ }^{36}$. Il unit pourtant les républicains de tous bords. À gauche, Eugène Bonnemère affirme dès 1856 : «La terre aux mains des paysans, c'est la vie même des sociétés livrée à la merci de l'impuissance, de la pauvreté, de l'ignorance la plus têtue et la plus persévérante ; c'est toute amélioration, tout progrès rendus impossibles ${ }^{37}$. Et chez les républicains proches des libéraux, c'est Ferry qui exprime sans doute le mieux les inquiétudes de son camp à l'égard de ces paysans si absorbés par la culture de leur parcelle qu'ils restent "indifférents " à tout progrès : "Quand la locomotive passe à toute vapeur, écrit-il en 1863, le paysan se lève sur le sillon, ses bras nus posés sur sa houe; son regard accompagne un instant le bruyant phénomène, puis lentement il recourbe son dos vers la terre. C'est de ce regard vague, rêveur et las, où se reflètent tant de misères, que le campagnard voit passer les plus grandes choses de ce monde. La liberté est de ce nombre. Comme le railway, elle lui est indifférente. ${ }^{38}$.

La concentration observée à la même époque dans le domaine industriel et financier ne fait qu'ajouter à la confusion : et si la petite propriété foncière n'était pas le signe du progrès mais au contraire un vestige du passé, susceptible de freiner le développement économique et social du pays et avec lui l'avènement de la République ? ${ }^{39}$

Il n'est pourtant pas question pour les républicains de condamner absolument la petite propriété foncière, qui reste à leurs yeux le seul moyen de se prémunir contre le retour de la féodalité terrienne mais aussi

36. Voir à ce sujet : Gilles PECOUT, «La politisation des paysans au XIX siècle. Réflexions sur l'histoire politique des campagnes françaises ", Histoire et sociétés rurales, $\mathrm{n}^{\circ} 2$, 1994, pp. 91-125; La politisation des campagnes au XIX siècle: France, Italie, Espagne, Portugal, Rome, Publications de l'École française de Rome, 2000.

37. Eugène BONNEMÈRE, Histoire des paysans depuis la fin du Moyen-Âge jusqu'à nos jours (1200-1850), précédée d'une introduction: an 50 avant Jésus-Christ - 1200 après Jésus-Christ, Paris, F. Chamerot, 1856, tome 2, p. 355.

38. Jules FERrY, La lutte électorale en 1863, Paris, E. Dentu, 1863, p. 10.

39. Une question analogue agite les républicains à propos des chemins de fer. Brunot MARNOT, "Les républicains devant la science et la technique : l'exemple des chemins de fer (1830-1937) ", in Paul BASQUIAT (dir.), Deux siècles de débats républicains (1792-2004), Paris, L'Harmattan, 2004, pp. 109-123. 
de résoudre la question sociale ${ }^{40}$. Bonnemère reconnaît ainsi que le morcellement du sol reste un "progrès", quoique "relatif " ${ }^{41}$, tandis qu'Alfred Mézières appelle les républicains à modérer leurs critiques: " $\mathrm{Ne}$ nous en plaignons pas. C'est une loi juste et inévitable qui s'accomplit. " ${ }^{42}$.

Mais de quelle loi s'agit-il ici ? D’une loi économique ou politique? Celle du marché ou du Code civil ? Rares sont les républicains qui répondent explicitement à la question, tant il est difficile pour eux d'admettre toute divergence entre le politique et l'économie, l'idéal révolutionnaire et les lois de l'évolution des sociétés. Pour l'heure, les républicains se contentent de chercher un remède susceptible de résoudre les contradictions du temps. Quand la plupart d'entre eux placent leurs espoirs dans le retour des libertés politiques bafouées par le régime impérial, certains évoquent déjà l'association, qui a longtemps eu les faveurs de la fraction la plus avancée du mouvement républicain : tout en respectant la petite propriété foncière, elle paraît à même de sortir les paysans de la stagnation économique et morale où ils végètent pour les amener à la fois à l'aisance et à la République ${ }^{43}$.

L'interrogation de Ferry face à la concentration industrielle "résister ou laisser-faire? " ${ }^{44}$ - suggère pourtant déjà le dilemme auquel les républicains seront ensuite confrontés sous la Troisième République : laisser-faire, comme les opportunistes, mais au risque d'être accusés de trahir la Révolution ; résister, comme les radicaux, mais au risque d'être accusés de craindre le progrès.

\section{Le défi du socialisme agraire}

Dans un siècle marqué par le positivisme, les hésitations républicaines autorisent toutes les critiques. Après les bonapartistes, qui sous le

40. Comme l'a montré Robert Castel, jusqu'à la fin du XIX siècle, seul l'accès universel à la propriété semble pouvoir résoudre la question sociale, le statut de nonpropriétaire ou prolétaire étant étroitement associé à l'insécurité. Robert CASTEL, Les métamorphoses de la question sociale : une chronique du salariat, Paris, Fayard, 1995.

41. R. CASTEL, Les métamorphoses..., op. cit., p. 356.

42. Alfred MÉZIÈrES, La société française: le paysan, l'ouvrier, la bourgeoisie, l'aristocratie, les femmes. Études morales sur le temps présent, Paris, Didier, 1869, p. 15.

43. Voir par exemple les auteurs cités par Eugène BONNEMÈRE, Le morcellement agricole et l'association, Batignolles, Hennuyer, 1859. L'ouvrage a d'abord paru sous la forme d'un article dans le Journal des économistes daté du 15 janvier 1859. p. 501 .

44. "La concentration industrielle", Discours et opinions de Jules Ferry, op. cit., 
Second Empire ont eu beau jeu de souligner la gêne de l'opposition républicaine et libérale face à la modernité $e^{45}$, ce sont des socialistes que viennent, au début de la Troisième République, les attaques les plus acerbes : les doctrines économiques des républicains les placeraient selon eux à contre-courant du progrès, quoi qu'ils en disent.

C'est tout l'enjeu du discours de Jaurès à la Chambre des députés en 1897 : démontrer que l'histoire, en imposant la mort inéluctable de la petite propriété foncière, va dans le sens du socialisme et discrédite au contraire les républicains, convaincus que l'heure est encore au morcellement du sol. Leur position témoigne selon lui d'une "méconnaissance des notions élémentaires du développement économique", qui les conduit à nier "l'histoire ": "Et comme ils barrent l'histoire dans le passé, par une sorte d'illusion qui leur fait transporter le présent dans les siècles disparus, en vertu de cette même illusion, ils barrent l'avenir ( $A p$ plaudissements à l'extrême gauche et sur divers bancs à gauche) et s'imaginent à tort immuable dans les siècles futurs ce qu'à tort ils considèrent comme ayant existé dans les siècles passés (Nouveaux applaudissements sur les mêmes bancs)" ${ }^{46}$.

Là encore, on peut s'interroger sur l'efficacité de son discours. La petite propriété foncière a en effet de beaux jours devant elle : dans la première époque de la III $^{\text {e }}$ République, note Maurice Agulhon, on «voit s'étendre (sinon vraiment triompher) cette démocratie de petits propriétaires qui, au lendemain de la Révolution française, tenait autant de l'espoir que des réalités " ${ }^{47}$. Trente ans avant la joute Jaurès/Deschanel, Marx en avait d'ailleurs déjà pris son parti. Dans la première édition du Dix-huit Brumaire de Louis Bonaparte, paru en 1852, il plaçait ses espoirs dans le mouvement de l'économie, qui porterait selon lui un coup fatal à la petite parcelle paysanne et détournerait dès lors les populations rurales de Napoléon III, en qui elles ne voyaient que le garant de leurs propriétés. Le paysan français, prolétarisé, n'aurait plus d'autre choix alors que

45. Un chapitre de ma thèse est consacré à cette question : Chloé GABORIAUX, Le paysan français, un enjeu idéologique au XIX siècle. Perspectives françaises et perspectives sur la France, thèse de science politique sous la direction de L. Jaume, IEP de Paris, 2008.

46. Séance du 26 juin 1897, Journal officiel de la République française, 27 juin 1897, p. 1693.

47. Maurice AGULHON, "Les transformations du monde paysan ", in G. DUBY, A. WAllon (dir.), Histoire de la France rurale, op. cit., p. 467. Voir aussi Claude SERVOLIN, "Les politiques agricoles", art. cit. ; et Jean-Luc MAYAUD, La petite exploitation triomphante..., op. cit. 
de rallier la cause de la classe ouvrière : "En désespérant de la restauration napoléonienne, le paysan français abandonne la foi en sa parcelle, renverse tout l'édifice d'État construit sur cette parcelle et la révolution prolétarienne obtient ainsi le chour sans lequel, dans toutes les nations paysannes, son solo devient un chant du cygne " ${ }^{48}$. Dans l'édition de 1869, Marx supprime pourtant cette dernière phrase : la petite propriété foncière semble résister, et avec elle le bonapartisme rural, ruinant ainsi le rêve socialiste d'une alliance prochaine de la paysannerie et du prolétariat. En 1897, alors que la France reste un pays de petite propriété, comment Jaurès pourrait-il convaincre lorsqu'il prédit l'« agonie de la petite propriété " ?

La question est pourtant biaisée, car elle donne aux faits économiques et sociaux une lisibilité qu'ils n'ont jamais eue. En favorisant dans certaines régions " une amorce de regroupement de la terre et de concentration des exploitations " ${ }^{49}$, la dépression de l'agriculture à la fin du XIX siècle a pu donner aux observateurs du moment le sentiment que la petite propriété foncière avait vécu ou du moins, se trouvait sérieusement menacée.

La position des républicains s'en trouve ébranlée. Non qu'ils hésitent à défendre la petite propriété foncière : ils y sont profondément attachés. Mais peuvent-ils encore la défendre non seulement comme une exigence républicaine mais aussi comme un fait inscrit dans le sens de l'histoire et du progrès ? C'est ce que Deschanel s'emploie à démontrer dans sa réponse à Jaurès, opposant les chiffres aux chiffres, les exemples de morcellement aux exemples de concentration. Mais lui-même semble parfois douter. La multiplication de la petite propriété foncière en France tientelle au cours naturel des choses ou à l'action volontariste des politiques ? Un passage de son discours, consacré à la comparaison de l'Angleterre et de la France, illustre bien l'incertitude de Deschanel. À propos de la domination de la grande propriété foncière en Angleterre, il affirme d'abord que «Marx s'est trompé en attribuant cette concentration terrienne à la marche normale des phénomènes économiques ». Elle est selon lui due à " une révolution essentiellement politique, et non [à] une évolution économique : la politique a violé, chez nos voisins, l'évolution économique ». On s'attend alors qu'il démontre que le morcellement du sol ob-

48. Karl MARX, Le dix-huit brumaire..., op. cit., p. 196, note 163.

49. Gabriel DÉSERT, "La grande dépression de l'agriculture", in G. DUBY, A. WALLON (dir.), Histoire de la France rurale, op. cit., p. 382. 
servé en France correspond au contraire à « la marche normale des phénomènes économiques ». Mais c'est pourtant un fait politique qu'il invoque, l'adoption en France du partage égal, inscrit dans le Code civil : "Aujourd'hui l'erreur n'est plus possible, la science a fait son ouvre, la lumière est faite. Il est avéré, il est constant que le Code civil, cette machine à hacher le sol, ne cesse de transformer la grande propriété en propriété moyenne et la moyenne en petite. " ${ }^{50}$

Deschanel n'hésite pas d'ailleurs à appuyer l'évocation de la statistique foncière par un argument non pas économique mais moral - non sans arrière-pensées électorales - qui condamne définitivement la description jaurésienne de la misère paysanne : "Salariés tenus en tutelle", "servitude", "état de demi inconscience, de passivité, de demi obscurité", "facultés hébétées par la continuité des privations", "existence à demi bestiale, à demi humaine" ah! qu'il entre d'orgueil dans vos jugements, et que votre pitié hautaine est voisine du dédain $!{ }^{51}$.

À un moment où le sens de l'histoire reste confus et à l'issue d'un demi-siècle qui a vu les conceptions républicaines de la petite propriété foncière souvent infléchies et remaniées, les incertitudes de l'argumentation suivie par Deschanel comme l'enthousiasme bruyant qu'elle a suscité dans les travées républicaines manifestent le véritable défi que constitue alors pour la République le socialisme agraire tel qu'il est présenté par Jaurès. Ce dernier retrouve en effet une cohérence que les républicains ont depuis fort longtemps perdue. Dans son discours, la marche du progrès économique est identifiée à la même loi de concentration, dans l'industrie comme dans l'agriculture, et converge en outre avec un idéal politique que Jaurès inscrit dans la continuité de la Révolution française. L'émancipation économique s'y conjugue avec l'émancipation politique et morale, dans un raisonnement qui reprend en les transformant les thèmes longuement développés par les républicains sous le Second Empire et au début de la Troisième République : « Le paysan étonné écoute et médite ; pour la première fois lui, l'égoïste et l'isolé, c'est par la longue souffrance des crises qu'il est entré en vivante communion avec la race humaine $"$.

50. Séance du 10 juillet 1897, Journal officiel de la République française, 11 juillet 1897, p. 1936.

51. Ibid., p. 1938.

52. Séance du 26 juin 1897, Journal officiel de la République française, 27 juillet 1897, p. 1692. 
Sans en avoir conscience sans doute, Deschanel atteste dans sa réponse l'attrait du discours de Jaurès : "Ce que le socialisme promet, affirme-t-il pour finir, l'association libre le tient ${ }^{53}$. Deschanel pense ici au mouvement syndical et coopératif, en plein essor depuis 1884, et que les républicains s'efforcent alors de soustraire à l'influence des grands propriétaires terriens, suspectés d'être réactionnaires. En le présentant comme la réalisation concrète des promesses socialistes, il suggère que la véritable divergence entre les républicains et les socialistes tient moins aux « faits " ou aux " affirmations générales » décrivant l'objectif à poursuivre $^{54}$ qu'aux moyens proposés pour y parvenir : collectivisation des terres ou association libre des petits propriétaires terriens.

Rapporter, comme on le fait trop souvent, la politique agricole des débuts de la Troisième République à des considérations politiques et idéologiques, c'est donc présupposer un rapport du politique à l'économique qui est justement sans cesse mis en question dans les débats ici étudiés. La défense de la petite propriété paysanne au cœur de la politique agricole cristallise en effet une interrogation majeure chez les républicains de la fin du XIX ${ }^{e}$ siècle : comment joindre les deux bouts de la chaîne des temps que les évolutions économiques, sociales et politiques du second XIX siècle ont contribué à dissocier - l'héritage de la Révolution française d'une part et la marche du progrès de l'autre? A la fin du $\mathrm{XIX}^{\mathrm{e}}$ siècle, on l'a vu, les républicains hésitent encore : pétris d'un positivisme qui les conduit à poser le sens de l'histoire comme extérieur à l'action humaine, ils ne parviennent pas à surmonter d'une façon qui leur paraisse totalement satisfaisante les contradictions qui travaillent leur idéal politique et social.

Chloé GABOrIaUX Docteur, Université Lyon 1

53. Séance du 10 juillet 1897, op. cit., p. 1944.

54. C'est ce sur quoi les deux orateurs prétendaient pourtant s'affronter. Ibid., p. 1933. 